\title{
A Quality Model for Evaluating IoT Applications
}

\author{
Mi Kim* \\ Department of Computer Science, Soongsil University, Seoul, Korea. \\ * Corresponding author. Email: pytwoori@gmail.com \\ Manuscript submitted October 24, 2015; accepted February 13, 2016. \\ doi: 10.17706/ijcee.2016.8.1.66-76
}

\begin{abstract}
In this paper, we focuses on suggestion to Quality model of IoT Applications which are popularly used in the characteristics defined in the quality model of IoT Applications. In order to achieve this purpose, we propose Quality model for IoT Applications. The IoT application is a complex fusion of a variety of technologies such as wireless network, embedded, sensor and connectivity. Because these technologies involves utilization and mobility in addition to quality characteristics in existing software, application of ISO 9126 is not perfect when evaluating IoT applications. It was considered to have some different views of attribute of the quality of service models in order to support convenient approach to modeling and IoT applications. We suggested new quality model for IoT applications by quality attribute in ISO 9126 . The effectiveness of the quality model for evaluating IoT application through scenario-based case studies were validated. The quality model for IoT application using the IA-QM proposed in this paper it can be measured relatively accurately.
\end{abstract}

Key words: QoS model, IoT applications, internet of things, quality attribute.

\section{Introduction}

Internet of Things(IoT) is the computing environment to provide valuable services by interacting with various IoT applications, where diverse devices are connected within the existing internet infrastructure and through intelligent social applications.

A Quality model for Software applications acts as a framework for the evaluation of attributes of an applications that contribute to the quality model. It is important every relevant software applications quality characteristic is specified and evaluated whenever possible using validated or widely accepted metrics.

It is necessity to customize a quality model to identify acceptance criteria and evaluate a particular application domain; IoT applications.

A natural consequence of the trends would be to manage the quality of IoT applications. However, measuring the quality of IoT applications is considerably different from measuring the quality of conventional software systems. This is mainly due to the characteristics of IoT devices and their applications which are not presented conventional software systems.

Because IoT application is a complex fusion of a variety of technologies such as wireless network, embedded, sensor and connectivity.

For instance, ISO 9126 which is a representative quality model is mainly for conventional software, not addressing the IoT characteristics. That is the quality attributes and metrics in ISO 9126 would largely inappropriate in measuring the quality of IoT applications.In this paper, we intend to propose a quality 
model to identify characteristics of quality factors which are quality attributes, criteria and metrics evaluate a particular IoT applications.

The rest of the paper is structured as follows: Related work in Section 2, characteristics of IoT applications is defined, in Section 3, quality attributes and metrics is defined, in Section 4. In next section, the proposed model will be applied for evaluation of a case study. Finally, a conclusion and future works are given in Section 6.

Fig. 1 shows that the service of IoT applications and each infrastructure is defined in this below.

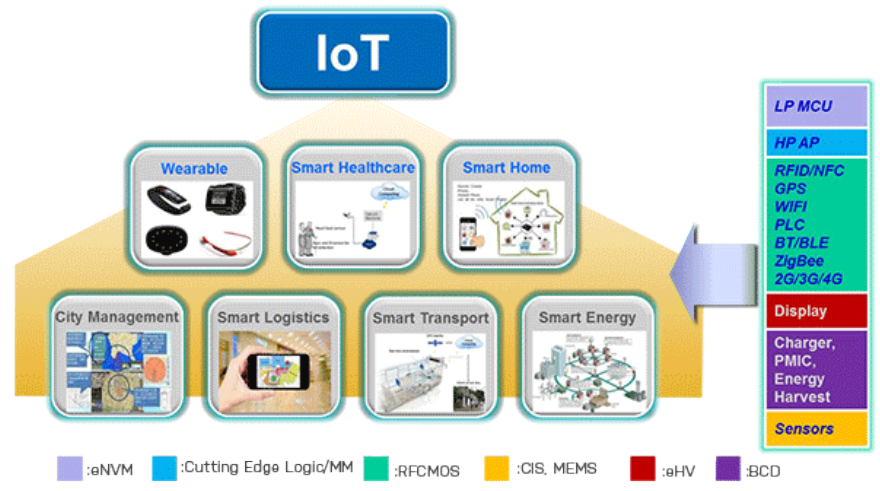

Fig. 1. Service of IoT applications.

\section{Related Works}

A Quality model has been studied by many researchers in conventional software. ISO/IEC 9126 is not perfect when quality of IoT applications. The Quality model can analyze IoT contexts and employ methods to compute the value of QoS which acts as a metric for service evaluation and selection. But most previous work focused on the RFID network protocols, middleware, devices reliability, safety and cost, etc.

Evan Welbourne deployed these applications in the RFID Ecosystem and conducted a four-week user study to measure trends in adoption and utilization of the tools and applications as well as users' qualitative reactions [1].

In [2], the author constructed another AHP QoS model based on IoT global etc. were considered but users' feedback like user preferences was often ignored.

Simple Performance Analysis of Multiple Access RFID Networks Based on the Binary Tree [3]. This simplifies tremendously the derivation of the analysis, still preserving its validity in an average sense. scalable RFID network simulator is developed with OPNET 11.0 and implements the binary tree collision arbitration protocol. The results obtained with simulator are very complexity.

Performance evaluation is conducted for tag read latency and read efficiency as a function of link parameters defined in ISO/IEC 18000-6 Type C standard [4]. But this ISO9126 is difference for application quality evaluation. Reference [5] first proposed a QoS model of grey decision-making from the view of IoT Global Infrastructure and built an adaptive service framework. In [6], a quality evaluation technique of RFID middleware according to ISO/IEC 9126 standard and EPC global middleware quality factors was built by simple AHP. It really provided an idea of simplifying complicated evaluation factors in IOT. ISO 9126 is an international standard for the evaluation of product quality [7]. This standard provides three aspects for evaluating software products; internal quality, external quality and quality in use. And, there are sixteen characteristics for three types of qualities. However, this standard focuses on evaluating quality of conventional products. Hence, it is required that the standard is customized and extended to evaluate the quality of IoT applications. 


\section{Charactersitics of IoT Applications}

IoT applications reveal non-conventional features which are not typically presented in conventional software systems. it is as in the following.

\subsection{Participation of Hardware Devices}

In IoT, things are required to be actively participating in various activities, exchanging information and making intelligent decision. IoT devices are equipped with various intelligent devices to ensure safety, security and high performance of IoT devices.

Mobile devices are equipped with small buffers but have to deal with IoT services that generate a huge volume of data

Hence, an IoT application consists of two types of elements; typical software components and hardware devices/components. In the design and implementation of IoT applications, the presence of hardware devices should be considered.

\subsection{Collaboration Model with IoT Devices}

A software application typically consists of multiple business processes, also called workflow, collaboration, orchestration. In IoT applications, collaborations could include the hardware functionality of IoT devices. For example, the Drone-based Parcel Delivery System by Amazon includes the parcel pick-up service and flying service of Drone devices.

The collaborations of an IoT application should consider the hardware functionality as well as conventional software collaboration.

\subsection{Mobility and Connectivity}

It refers to the application capability for device mobility the correctness of the information processing in different stages of a process. This characteristic is merely used in the web application and particulary in IoT applications.

Connectivity characteristics refers to the ease of user's quick and efficient connect to information of IoT applications.

Many of IoT devices have mobility; either extrinsic mobility by human users such as Healthcare Smart Watch or intrinsic mobility by built-in moving mechanisms such as AR Drone. The mobility could result in various faults; loss of control due to out-of-sight movement or flight, unstable wireless network connection when leaving the current network zone, and physical collision while moving.

\subsection{Monitoring for IoT devices}

Remote monitoring for smart devices attached to networks can support multiple functions including Automatic Meter Reading, gas heating and context-awareness.

Through smart devices, carriers can provide more real-time and precise services.

\subsection{Limited Resources}

The resource types of IoT devices can be battery, network communication facility, memory, and computation power. For example, flight Drone has a limit battery and memory, leaving the current network zone.

IoT devices suffer from a limited battery lifetime and dominate energy consumption. Energy Efficiency can be increased by wisely adjusting transmission power.

Hence, there is a need for solutions that will limit the energy consumptions of such IoT devices such as wearable device, smart watch.

Fig. 2 shows that the characteristics of IoT applications and each criterion is defined in this section. 


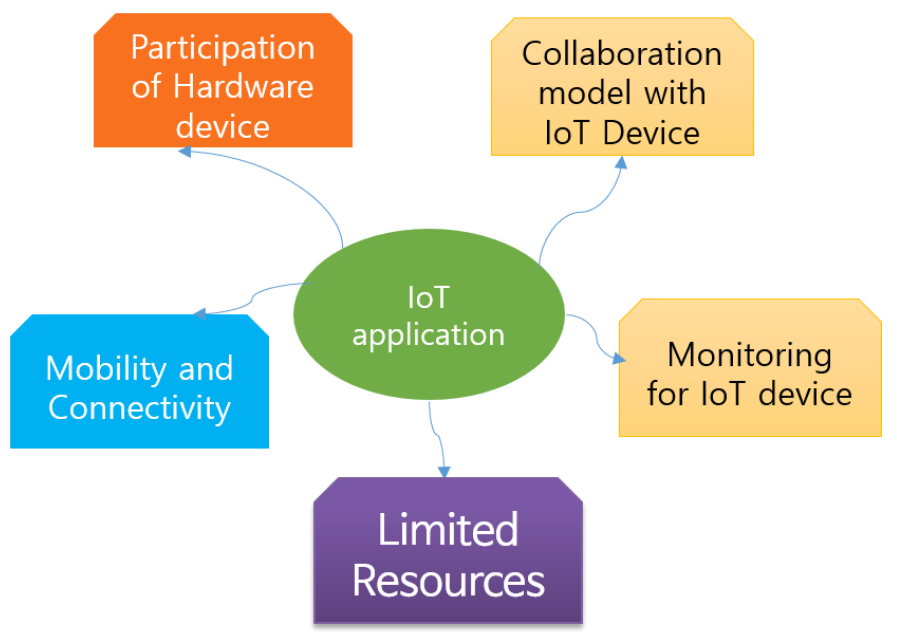

Fig. 2. Characteristics of IoT applications.

\section{Quality Attributes and Metrics}

The characteristics defined in the previous section become the basis for deriving the quality model of IoT applications. That is, we define quality attributes for evaluating IoT applications by considering the impacts of the identified characteristics on the quality of IoT applications.

\subsection{Quality Attributes for Evaluating IoT Applications}

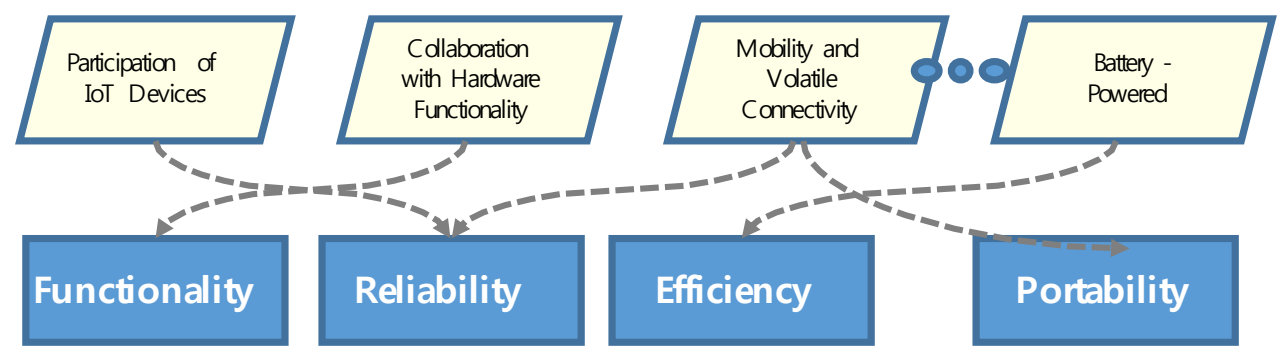

Fig. 3. Mapping characteristics to quality attributes.

Each characteristics of IoT applications has a set of associated quality factors. Fig. 3 shows how the quality att ributes of the evaluating IoT applications affect the selection of quality factors.

Functionality: The functionality is required to interoperate across interconnected IoT sensor device, means that the interoperability for the IoT device chips connected other things. In case of IoT applications, the functional requirement is defined in characteristics of IoT applications requirements specify particular results of IoT applications. For example, the hardware functionality of In some cases, there may drone based parcel delivery system and flying service of drone devices. The collaborations of an IoT applications should consider the hardware functionality as well as conventional software collaboration. The functionality of IoT applications should be measured differently from conventional measurement of software functionality.

Sub-factors for Functionality: Suitability (functional coverage), Accuracy (for supported function, how accurate it?)

Suitability means that the functionality of the applications fits the needs of a user to fulfill a certain task on the IoT applications. Accuracy means applications provided by IoT applications that can correctly execute the various specified instructions from customers needs data in order to them.

Suitability is an external suitability metric measure an attribute such as the occurrence of an unsatisfied operation during testing and user operation of the IoT applications. 
Security is the ability to prevent unauthorized access, whether accidental or deliberate, to programs and data. Also wearable computing is consists of minimal chips, to prevent the network applications sometimes from physical stolen.

The security problem is an important quality characteristic as the elements has significant impact on the user's reliability.

The combination of hardware and software functions differs from traditional software functionality.

Interoperability means that the application is able to interact with the specified systems through sensor network and IoT device such as minimal chips.

Collaboration means that the in IoT applications, collaborations could include the hardware functionality of IoT devices.

Compliance means that the application is compliant with standards, conventions or regulations in laws and similar prescriptions to IoT applications. Collaboration on IoT applications is an insufficient Therefore, standard of the quality characteristics is very important factor.

Reliability: The reliability is the capability of a measuring specification to maintain a specific level of performance each IoT applications and sensor devices which are different conditions. The context data can makes status of the environment data and provides a service of data. The reliability of the fits the needs of a user to execute a specific task on the IoT applications.

Maturity is the frequency of software faults to deal with software faults or next fail, its specified interface.

The capability of data recovery expresses the performance that using the minimal chips gathering the data acquisition.

The acquisition data test results should always be reproducible in subsequent test runs if generally possible. Maturity is the frequency of software faults.

Sub-factors for Reliability: Fault tolerance is the ability of software to deal with of software faults or infringement of its specified wearable computing as IoT applications.

Fault Tolerance - on faults, minimum performance should be delivered.

Recoverability - when failed, how effectively the system can be recovered. Because it provides services to meet the requirements, when a fault occurring, recoverability in case of defects is an important quality factor.

Connectivity characteristics refers to the ease of user's quick and efficient connect to information of IoT applications. In this context, the word "performance" expresses the degree to which needs are satisfied. It refers to the application capability for device mobility the correctness of the information processing in different stages of a process. The reliability is mainly factor of IoT applications that the capability correctness for context awareness data processing has subcharacteristics maturity, fault-tolerance, and recoverability of ISO 9126 apply to test specifications as well.

Mobility is requires developing a usability over network to be carried out in IoT device networks with high specification related with low power consumption and frequent data moving capacity. The RFID/NFC technologies need to support secure connection to IoT applications, Our approach is draw a quality attribute standard and metrics by identifying quality characteristics.

Efficiency: The efficiency characteristic relates to the capability of a test specification to provide acceptable performance in terms of speed and resource usage. The efficiency of the time and resource behavior is distinguished.

IoT devices suffer from a limited battery lifetime and dominates energy consumption. Energy Efficiency can be increased by wisely adjusting transmission power.

Hence, there is a need for solutions that will limit the energy consumptions of such IoT devices.

Sub-factors for Efficiency: The time behavior describes for instance processing times and throughput 
rates while resource behavior means the limited battery and memory as amount of resource used and the duration of use. Hence, Efficient resource utilization, according to resource efficiency because it may show a difference in processing time, throughput is an important quality factor.

Portability: Portability is capability of change another environment system, installability (ease of installation in a specified environment), co-existence (with other test products in a common environment), and replaceability (capability of the product to be replaced by another one for the same purpose) are too concrete. However, adaptability is relevant since test specifications should be capable to be adapted to different IoT product as a flexible display, battery or augmented reality implementation environments. For example, network computing, wearable computing and sensory data access to specification make it hard to adapt the specification for other network chips.

Sub-factors for Portability: Adaptability is the opportunity for adapting the application to different environments without additional effort.

Installability is the effort for installing the software Conformance means the conformance of an application to standards or conventions relating to portability.

Replaceability is the opportunity and effort to use an application as a replacement for another IoT applications.

Sustainability is the resource types of IoT devices can be battery, network communication facility, memory, and computation power by wearable device is limited. The Wearable Computers augmented reality is limited a high-end to the ultra-compact, low-power devices by frequency of use of the image representation technique and movement of the data which are many battery consumption. Hence, A lot more battery power, once the restrictions on the portability and practicality of wearable computer draw a sustainability quality attributes.

Fig. 4 shows the sub factor characteristics to quality attributes, and each criterion is defined in this section.

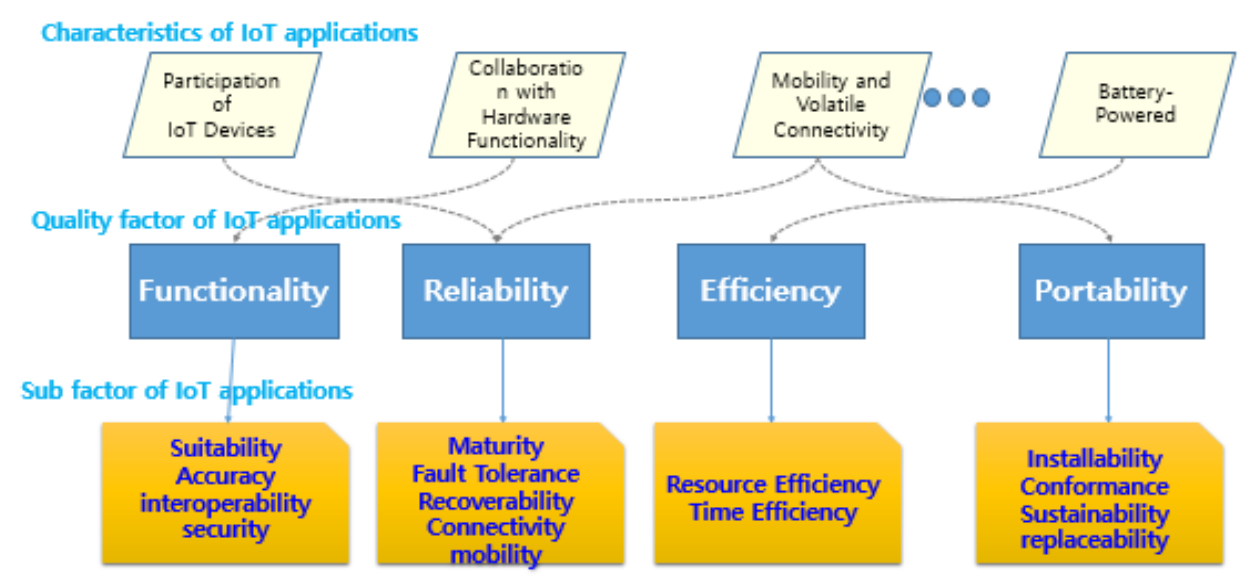

Fig. 4. Sub-factor characteristics to quality attributes.

\subsection{Metric Suite for the Quality Model}

In this section we provide a set of representative metrics for the criteria like all quality models, the set of proposed metrics here may be extended or tailored for each organization. Hence, the proposed set of attributes should serve as the foundation and a starting point for tailoring and enhancement.

\subsubsection{Metrics for functionality}

Metric for Functionality: this metric measures the degree of functional connection to IoT applications, as follows. 
$X=A / B$, where $A$ is the number of correctly accessibility related to functionality of characteristics for IoT application is applicable to, and $B$ is the total number of functional accessibility in the IoT domain.

The Range of $X$ is 0.1 , and the value 1 is the best since the functionality of such a application can be accessed to all functional items in the IoT domain. The higher the metric value has wider range of accessibility.

Metric for Suitability: The metric measures the scope of functionality provided by a user operation fits of the IoT application as follows:

$X=A / B$, where $A$ is the number of input items which variety environment system of user satisfied requirements, and $B$ is the total number of functions required to be satisfied items which can be obtained from IoT applications.

The Range of $X$ is $0 . .1$, and the value 1 is the best since the such a functions and implement and provides all required service. The metric can be applied to a set of functions.

Metric for Interoperability: The metric measures how the interact with the specified systems through network application as follows:

$X=A / B$, where $A$ is the number of fulfillment each peripheral which failed performance, and $B$ is the number of performance which can be required by the user.

The Range of $X$ is $0 . .1$, and the value 1 is the best since the such a functions and performance each facility which obtained IoT applications network.

Metric for Security: The metric measures how the safety from the network infringement through IoT applications are.

$X=A / B$, where $A$ is the number of access authority failed which can be safety during system operation, and $B$ is the number of entire authority request number.

The Range of $X$ is $0 . .1$, and the value 1 is the best. The close to 1 is higher authority. It can be increased user requirements.

\subsubsection{Metrics for reliability}

Metric for Reliability: The metric measures how the without seamless each IoT application connection as follows:

$X=A / B$, where $A$ is the number of input items which check for valid data, and $B$ is the number of total input items which could check for valid data.

The Range of $X$ is $0 . .1$, and the value 1 is the best. Although practically data will have a value that is high due to validation data.

Metric for maturity: The metric measures how the software faults to deal with next fail through IoT application as follows:

Frequency of system failure (failure rate)

$X=A / B$, where $A$ is the number of frequency of system failure, and $B$ is the number of presence of faults which can be occurring software bugs for validation data.

The Range of $X$ is $0 . .1$, and the value 1 is the best since such a error free systems all variable features needed. A metric for measuring the effectiveness is on our list of future research topics.

Metric for recoverability: The metric measures how Allowing users to take corrective action once an error has been recognized by the system.

$X=A / B$, where $A$ is the number of functions having status recovering quickly from a failure, and $B$ is the number of functions that are error handling, resuming work soon after a failure.

The Range of $X$ is $0 . .1$, and the value 1 is the best since such a self-control. However, applications with the value 1 will be a typical since most applications will have some degree of recovering for systems.

\subsubsection{Metrics for efficiency}


Metric for efficiency: The metric measures the efficiency appropriate time and resource behavior.

$X=A / B$, where $A$ is the time of limited battery utilization, and $B$ is the time of user requirement time and resource.

The Range of $X$ is $0 . .1$, and the value 1 is the best, The time and resource utilization describes for instance processing times and throughput rate, while resource behavior means the amount of resource used and the duration of use.

\subsubsection{Metrics for portability}

Metric for conformance: The metric measures the degree of change to relevant environments.

$X=A / B$, where $A$ is the number of system response request which can be customized, and $B$ is the user expected response requirements number can be changed relevant environment system as a sensory data creative value in the IoT applications.

The Range of $X$ is $0 . .1$, and the value 1 is the best, response request number for heterogeneous system such as a sensory data through IoT applications is higher for system utilization

\subsubsection{Sum of IoT applications metrics}

Using the proposed a set of factors and criteria, one can compute the overall quality of a IoT applications as follows. Let $F_{i}$ be the ${ }_{i}$ th factor defined in IA-QM, and therefore the range of $i$ is $1 . .4$ Let $C_{i j}$ be the ${ }_{j}$ th criterion of the $F_{i}$, and $F_{i}$ has 2 to 4 criteria as shown in Fig. 4.

For example, $C_{2,3}$ will be the customizability criterion for the reliability factor. Then the composite value, $Q$ $F_{i}$ for the factor $F_{i}$ is defined as:

$$
Q F_{i}=\frac{\sum_{j=1}^{n} C i j}{n}
$$

where $n$ is the number of criteria in $F_{i}$

Depending on the domain and purpose, often different weight on $F_{i}$ and let $(\mathrm{W} 1+\mathrm{W} 2+\mathrm{W} 3+\mathrm{W} 4)$ be equal to 1 .

Then, the overall quality of a functions can be computed as follows, and the range of overall quality will be 0.1 , which is same as a single percentage representation.

$$
\mathrm{IA}-\mathrm{QM}=\sum_{i=1}^{4}(w i \times Q F i)
$$

In order to apply the proposed IA-QM effectively, a quality evaluation process, instruction and a set of templates should also be defined.

As shown in Table 1, Weight for the quality attributes such as high (0.3), medium (0.2) and low (0.1) would be represented.

Table 1. Weight for the Quality Attributes

\begin{tabular}{|c|c|}
\hline Quality attributes & Weight \\
\hline Functionality(Func) & Medium (0.2) \\
\hline Reliability(Rel) & Medium (0.2) \\
\hline Efficiency(Eff) & Medium (0.2) \\
\hline Portability(Por) & Medium (0.2) \\
\hline SUM & 1 \\
\hline
\end{tabular}

\section{Case Study and Evaluation}


To show the applicability of the proposed quality model, we conduct a case study of applying the quality model on an IoT application.

\subsection{IoT Application under the Case Study}

In this section, IoT applications create a virtual environment to demonstrate the validity of the matric is applied to verify the viability of the scenario (see Table 2).

Table 2. User Kim's Scenario

\begin{tabular}{|c|c|}
\hline $\begin{array}{c}\text { Service } \\
\text { Environment }\end{array}$ & Scenario \\
\hline Requirement & $\begin{array}{l}\text { We perform a case study of a using IoT application to verify functionality on the scenario. } \\
\text { User Kim is the on / off and brightness adjustment the lights in the house using a smartphone. } \\
\text { User Kim Identify the ingredients, check the fridge before user going home. }\end{array}$ \\
\hline Mart & $\begin{array}{l}\text { Using a Map service confirms the market place. } \\
\text { It took time to find the position of two seconds. Memory usage time is spent batteries were used for } \\
7.8 \text { seconds and } 10 \% \text {. } \\
\text { Mart to buy the necessary ingredients to stop by using a smartphone to check your mail. It took } 2 \\
\text { seconds to access the e-mail. } \\
\text { To relax while watching a DMB was spent } 3 \text { second to float on the screen upon request. }\end{array}$ \\
\hline Navigation & $\begin{array}{l}\text { By using the Smart Watch to identify the location to avoid the rush hour to go home. } \\
\text { To connect the smart watch was spent two seconds. }\end{array}$ \\
\hline Home & $\begin{array}{l}\text { User kim has to search for recipes with a smart watch to get home. The wait time was about two } \\
\text { seconds. } \\
\text { Use the Smart Watch to make sure that today the product is sold, how the electronic oven to cook } \\
\text { while you wait Put. } \\
\text { Due to deadlines, the server was down } 30 \text { seconds were again operating normally. } \\
\text { Service response was } 5 \text { seconds was spent the used memory is } 4 \text { seconds. } \\
\text { Preference share purchases and sales per customer through the ages smart display, product-specific } \\
\text { and sex-buying status checks at a glance. } \\
\text { It took } 8 \text { seconds to pops up the statistics on the Smart Display. } \\
\text { Make sure Kim's child comes back to the smart location based applications. The time it takes to } \\
\text { determine the location is about } 3 \text { seconds. }\end{array}$ \\
\hline Exercise & $\begin{array}{l}\text { Smart treadmills and jogging as it takes time and mileage during exercise and calculates the calories } \\
\text { burned. } \\
\text { Service wait time is about } 5 \text { seconds, and the time was used three times } 38 \text { seconds. }\end{array}$ \\
\hline
\end{tabular}

\subsection{Results of the Case Study}

In this section, we showed that the proposed model can be practiced it was applied to evaluate the matric through the Case Study.

For the functionality calculated by applying the Kim' scenario defined in Section 4.1.

To using the number of total service is 8th applying in the scenario.

In this paper, we calculated for the functionality. $A$ is the number of success accessibility for 6 th and $b$ is the total number of accessibility for 8th.

$$
\text { Func }=\left(\frac{7}{8}\right)\left(\frac{\text { the number of success accessibility }=7}{\text { the total number of functional accessibility }=8}\right)
$$

Consequently, functionality is 0.875 , the value is close to 1 the functionality is high represented since such 
error free systems all variable features in the Kim's scenario.

To calculate the service of validation data applying the Kim's scenario.

For the Reliability calculated by applying the Kim's scenario defined in Section 4.1.

In this paper, we calculated for the Reliability. $A$ is the number of valid data for 7 th and $b$ is the total number of valid data for 8 th.

$$
\operatorname{Rel}=\left(\frac{7}{8}\right)\left(\frac{\text { the number of valid data }=7}{\text { the total number of valid data }=8}\right)
$$

Consequently, Reliability is 0.88 , the value is close to 1 the reliability is higher represented that the IoT Service is higher reliability.

To calculate the service of validation data applying the Kim's scenario.

For the Efficiency calculated by applying the Kim's scenario defined in Section 4.1.

In this paper, we calculated for the Reliability. $\mathrm{A}$ is the time of limited battery utilization time is 7.8 seconds, and B is the time of user requirement time and resource are 8 seconds.

$$
\text { Eff }=\left(\frac{7.8}{8}\right)\left(\frac{\text { the limited battery utilization time }=7.8}{\text { user expected request time }=8}\right)
$$

Consequently, Efficiency is 0.975, the value is less than expected time, the Efficiency is higher represented that the IoT Service has realized higher Efficiency.

For the Portability calculated by applying the Kim's scenario defined in Section 4.1.

In this paper, we calculated for the Portability. A is the number of user response request and B is the number of user expected response request.

$$
\text { Por }=\left(\frac{7}{8}\right)\left(\frac{\text { the number of system response request }=7}{\text { user expected response request }=8}\right)
$$

Consequently, Portability is 0.875 , the value is close to 1 the Portability is higher represented that the Portability is higher represented that the IoT Service higher Portability.

As shown in Table 3, Weight for the quality attributes such as high (0.3), medium (0.2) and low (0.1) would be represented.

For convenience and to calculate the weight to 0.2 applied sum of IoT applications metrics in phrase 5 as follows. As shown in Table 3, the value of IA-QM is 0.72 and the more high IoT application service because it is close to the Kim's scenario, the quality is relatively high.

Table 3. Values of IA-QM

\begin{tabular}{|c|c|c|}
\hline Quality attributes & Weight & Sum \\
\hline Functionality(Func) & $0.875 \times 0.2$ & 0.175 \\
\hline Reliability(Rel) & $0.88 \times 0.2$ & 0.176 \\
\hline Efficiency(Eff) & $0.975 \times 0.2$ & 0.195 \\
\hline Portability(Por) & $0.875 \times 0.2$ & $\mathbf{0 . 7 2}$ \\
\hline
\end{tabular}

As shown in Table 4 compares the items that can be separated by a quality measure above a support ( 0 ), partial support $(\Delta)$ Not supported $(\times)$. 
Table 4. Comparison of Existing Research

\begin{tabular}{|c|c|c|c|}
\hline Evaluation Item & Grey & AHP & IA-QM \\
\hline What is the procedure for a quality property presents exactly? & o & o & o \\
\hline What quality attributes are well defined? & $\circ$ & $\Delta$ & ○ \\
\hline What metrics are well defined in order to measure the target? & $x$ & $x$ & ० \\
\hline $\begin{array}{l}\text { What metrics the description for the quality measurements and metrics for } \\
\text { official Is the range of values been defined? }\end{array}$ & $\Delta$ & $\Delta$ & o \\
\hline
\end{tabular}

\section{Conclusion}

In this paper, we showed identified main characteristics of IoT applications, and derive a practical quality model for IoT application, IA-QM. The IoT application is a complex fusion of a variety of technologies such as wireless network, embedded, sensor and connectivity. Because these technologies involves utilization and mobility in addition to quality characteristics in existing software, application of ISO 9126 is not perfect when evaluating IoT applications. We suggested new quality model for IoT applications by quality attribute in ISO 9126. We defined four quality factors, criteria, and four metrics(Func, Rel, Eff, Por) that can be effectively used to derive the overall quality of IoT applications. The effectiveness of the quality model for evaluating IoT application through scenario-based case studies were validated. The quality model for IoT application using the IA-QM proposed in this paper it can be measured relatively accurately.

\section{References}

[1] Welbourne, E., Battle, L., Cole, G., Gould, K., Rector, K., Raymer, S., et al. (2009). Building the internet of things using RFID: the RFID ecosystem experience. IEEE Internet Computing, 13(3), 48-55.

[2] Fan, S. S., Shi, W. X., Wang, N., \& Liu, Y. (2011). MODMbased evaluation model of service quality in the internet of things. Procedia Environmental Sciences, 11, 63-69.

[3] Ferrari, G., Cappelletti, F., \& Raheli, R. (2008). Simple performance analysis of multiple access RFID networks based on the binary tree protocol. International Journal of Sensor Networks, 4, 194-208.

[4] Ko, C., Roy, S., Smith, J. R., Lee, H. W., \& Cho, C. H. (June 2008). RFID MAC performance evaluation based on ISO/IEC 18000-6 Type C. IEEE Communications Letters, 12(6).

[5] Liu, J. H., \& Tong, W. Q. (2010). Adaptive service framework based on grey decision-making in the internet of things. Proceedings of 2010 6th International Conference on Wireless Communications, Networking and Mobile Computing.

[6] Oh, G., Kim, D. Y., Kim, S. I., \& Rhew, Y. (2006). A quality evaluation technique of RFID middleware in ubiquitous computing. Proceedings of International Conference on Hybrid Information Technology: Vol. 2 (pp. 730-735).

[7] Software Engineering — Product Quality, Part 1: Quality Model. (June 2001). ISO/IEC 9126.

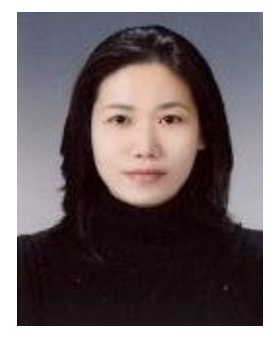

Mi Kim received the bachelor and master degrees from University of Honam, in 2000 and 2002, respectively. She is a candidate for the $\mathrm{PhD}$ degree at the Department of Computer Science at Soongsil University in Seoul, Korea. She had worked in LG Electronics Inc. in 1996 and 1999 and now she is a project manager and programmer at the IT Solution Cor. Hers research interests include software engineering, IoT applications, software quality. 